\title{
Persistently high levels of immune activation and their correlation with the HIV-1 proviral DNA and 2- LTR circles loads, in a cohort of Mexican individuals following long-term and fully suppressive treatment.
}

\section{Aurelio Orta-Resendiz}

Instituto Nacional de Ciencias Médicas y Nutrición Salvador Zubiran https://orcid.org/0000-00026632-883X

\section{Monica Viveros-Rogel}

Instituto Nacional de Ciencias Medicas y Nutricion Salvador Zubiran

Luis L Fuentes-Romero

Instituto Nacional de Ciencias Medicas y Nutricion Salvador Zubiran

\section{Moises Vergara-Mendoza}

Instituto Nacional de Ciencias Medicas y Nutricion Salvador Zubiran

\section{Damaris P Romero-Rodriguez}

Instituto Nacional de Enfermedades Respiratorias

\section{Monica Munoz-Lopez}

Instituto Nacional de Ciencias Medicas y Nutricion Salvador Zubiran

\section{Martha L Zancatl-Diaz}

Instituto Nacional de Ciencias Medicas y Nutricion Salvador Zubiran

\section{Elsa Y Vidal-Laurencio}

Instituto Nacional de Ciencias Medicas y Nutricion Salvador Zubiran

\section{Roberto A Rodriguez-Diaz}

Instituto Nacional de Ciencias Medicas y Nutricion Salvador Zubiran

Luis E Soto-Ramirez ( $\sim$ lesoto@hotmail.com)

\section{Research}

Keywords: HIV reservoir, proviral DNA, 2-LTR circles, immune activation, chronic cART

Posted Date: January 24th, 2020

DOI: https://doi.org/10.21203/rs.2.21753/v1

License: (1) This work is licensed under a Creative Commons Attribution 4.0 International License. Read Full License 
Page $2 / 23$ 


\section{Abstract}

Background The main cause of the persistently high levels of immune activation in HIV positive patients undergoing suppressive chronic cART is still unknown. Previous findings have suggested a link between ongoing residual viral replication originating from the HIV reservoir and the immune activation levels. However, there is no clear evidence of this assumption. The aim of this study was to investigate the correlation between the reservoir and the levels of immune activation in chronic patients under fully suppressive cART.

Methods We conducted a prospective longitudinal study in a cohort of HIV positive patients undergoing CART for more than 5 years without any documented blips. We quantified the HIV proviral DNA and the 2LTR circles loads from PBMCs, the levels of immune activation and proliferation markers of T-cells (CD38+, Ki-67+), and the levels of plasmatic IL-7 at enrollment and 1-year of follow-up. Correlation analysis and group comparison were performed.

Results 29 participants with a median of 8 years (IQR, 6.9-9.4) under suppressive cART were enrolled and successfully followed at 1 year. In this cohort, we found higher levels of CD8+ T-cell activation (CD38+) after 1-year $(P=.000)$. There was a very weak correlation between the levels of immune activation and the proviral DNA of CD4+ T-cell and CD8+ T-cell. The levels of Ki-67+ T-cells declined on time without significant differences, and there was no significant correlation with the proportion of activated T-cells. The plasmatic levels of IL-7 decreased at the follow-up observation $(P=.094)$, but there was no correlation with the levels of immune activation either.

Conclusions We found a weak correlation of the levels of CD4+ and CD8+ T-cell activation with the proviral DNA and 2-LTR circles. This suggests the likely occurrence of further mechanisms driving chronic versus early immune activation other than viral replication by itself in subjects under chronic suppressive CART. More importantly, we highlight the relevance of decreasing T-cell activation in chronic patients to lower the risk of morbidity and early mortality by investigating other activation pathways in specifically chronic phases.

\section{Background}

Combined antiretroviral therapy (CART) is the milestone treatment for HIV infection and AIDS. CART has demonstrated to be the most effective treatment that can successfully control the levels of viral replication, as clinically determined by maintaining viraemia under the levels of detection on routine and follow-up testing. Yet, even in the presence of effective treatment, highly abnormal levels of immune activation can be detected in HIV-1 positive patients, a phenomenon that has been associated with disease progression and higher levels of morbidity as compared with those of the general population (1$5)$.

The control of abnormally high levels of immune activation is crucial to prevent morbidity, especially in chronic patients under CART. 
The viral and immunological mechanisms driving immune activation are not completely understood, and the main cause has not been established. Some of the proposed potential causes are; the HIV replication by itself, the loss of mucosal integrity and microbial translocation, the immune response to other concurrent infections and the altered balance of CD4 + T-cell subsets among others (6-11). On the other hand, it has been proposed that immune activation in HIV positive patients could potentially have different origins in the early versus chronic phases of the infection.

Data showing the increase of immune activation markers rising from acute HIV infection has directed to consider that it could be directly triggered or caused by the presence of viral replication $(6,12)$.

Also, it has been described in previous findings an association between the viraemia and the levels of activation of CD8 + T-cells in the absence of treatment (13). Moreover, data shows that immune activation decreases following initiation of CART, but it fails to return to levels found in HIV negative subjects $(12,14)$. Therefore, it has been suggested a potential association between the levels of immune activation and residual viral replication originating from the reservoir despite effective cART. Furthermore, higher levels of activation have been linked to transient viraemia and blips, which suggests that ongoing residual replication originating from the HIV reservoirs could be driving immune activation despite the presence of CART $(15,16)$. However, there is no clear evidence to support this association, particularly in subjects undergoing chronic cART.

Data shows that a larger reservoir is responsible for proportionally more viral replication and a faster rebound of the viral load in patients stopping CART $(17,18)$. The detection of episomal 2-LTR circles is commonly accepted as a marker of ongoing viral replication, while the quantification of HIV-1 proviral DNA from peripheral blood mononuclear cells (PBMCs) has been described as a representative marker of the size of the reservoir in the human body.

This study aimed to assess the correlation between the immune activation levels and the reservoir in a cohort of HIV-1 infected subjects undergoing chronic cART with fully suppressed viraemia.

\section{Material And Methods}

\section{Study design}

We conducted a prospective study including HIV-1 infected patients attending the HIV clinic of the Infectious Diseases Department at the Instituto Nacional de Ciencias Médicas y Nutrición Salvador Zubirán (INCMNSZ) in Mexico City between January-August of 2018. Subjects over 18 years of age, under long-term suppressive cART (defined as HIV-1 RNA viral load measurements $<50 \mathrm{copies} / \mathrm{mL}$ by commercial assays without documented blips during at least 5 years of follow-up) and currently on their first CART regimen were included to be followed after 1 year. Enrolled participants also had to have no comorbidities, nor documented co-infections in their clinical record, or any other active disease for at least two months prior to inclusion. We collected in a database the age, birth sex, cART regimen, HIV RNA viral load history, CD4 + T-cell count nadir and CD 4 + and CD $8+$ T-cell count at inclusion and follow-up visits. 
The laboratory testing for the HIV-1 proviral load (proviral DNA), 2-LTR circles, markers of activation and proliferation (CD38 + and Ki-67+), as well as interleukin 7 (IL-7) were performed after screening and at one year of follow-up. The study was approved by the research and bioethics boards of the INCMNSZ, and was conducted in accordance with the Helsinki Declaration. All participants provided written informed consent prior to recruitment.

\section{Laboratory assays}

Plasma was obtained by centrifugation and PBMCs were isolated by density gradient media from blood samples collected from the patients. HIV-1 proviral DNA was measured by Real-time PCR and 2-LTR circles were measured by SYBR Green Real-time PCR. Immune activation CD38 + and proliferation Ki-67 + biomarkers were analysed in PBMCs by flow-cytometry and plasma IL-7 levels by a commercial ELISA assay. Complete laboratory methods are detailed in the supplemental material.

\section{Statistical analysis}

Data analysis was performed using descriptive and central tendency statistics expressed as median and inter-quartile range (IQR). For certain analysis, subjects were stratified in sub-groups: by years under suppressive cART ( $\leq 10$ and $>10$ years) and by type of third cART-component (Protease inhibitor [PI] and non-nucleoside reverse transcriptase inhibitor [NNRTI]). We employed the non-parametric Spearman correlation test for quantitative variables, the Wilcoxon-signed rank test and the Mann-Whitney $U$ test for independent groups comparisons with the PRISM v7 software (GraphPad Software Inc., La Jolla, CA, USA). A P $<.05$ was considered statistically significant.

\section{Results}

\section{Study population}

A total of 104 patients were evaluated for eligibility. Of these, 38 participants were first enrolled. A total of 9 subjects were excluded from the study after 1 year of follow-up: 4 failed to attend the follow-up visit, 2 were excluded due to an HCV infection of recent diagnosis and 3 for incomplete data. Pre-treatment and baseline characteristics of the participants are summarized in Table 1. Our participants had a median age of 47 years at recruitment and most of them were men. The median pre-treatment viral load (plasma HIV RNA) was $5 \log _{10}$ copies/mL (IQR, 4.9-5.2 $\log _{10}$ copies/mL) and the nadir of CD $4+$ T-cell count was 119 cells $/ \mathrm{mm}^{3}$ (IQR, 31.5-266 cells $/ \mathrm{mm}^{3}$ ). Most participants were under a cART regimen based on a NNRTI (89.7\%), while the others were on a PI-based regimen (10.3\%). 
Table 1

Pre-treatment and baseline features

\begin{tabular}{|c|c|c|}
\hline Feature & Median & IQR \\
\hline Age at enrollment (years) & 47 & $41.5-52$ \\
\hline Age at HIV diagnosis (years) & 36 & $30.5-43$ \\
\hline Time under cART at enrollment (years) & 8 & $6.9-9.4$ \\
\hline Pre-treatment viral load $\left(\log _{10} \mathrm{c} / \mathrm{mL}\right)$ & 5 & $4.9-5.2$ \\
\hline Nadir of CD $4+$ T-cells $\left(\right.$ cell $/ \mathrm{mm}^{3}$ ) & 119 & $31.5-266$ \\
\hline Pre-treatment CD4 + T-cell count $\left(\right.$ cell $\left./ \mathrm{mm}^{3}\right)$ & 168 & $44.5-271$ \\
\hline Pre-treatment CD8 + T-cell count $\left(\right.$ cell $\left./ \mathrm{mm}^{3}\right)$ & 785 & $377-1128.5$ \\
\hline Pre-treatment $\mathrm{CD} 4 / \mathrm{CD} 8$ ratio & 0.19 & $0.09-0.34$ \\
\hline IQR, interquartile range; $\mathrm{N}=29$. & & \\
\hline
\end{tabular}

As expected, due to cART efficacy, we observed in our participants a significant recovery of the CD4 + Tcell as shown in Fig. 1A. Likewise, we also observed an increase of the CD4/CD8 ratio and a slight increase of the CD8 + T-cell count at follow-up compared to that at enrollment (Fig. 1B and 1C).

\section{HIV proviral DNA and 2-LTR circles}

To study the size of the HIV reservoir, we were able to measure the HIV proviral DNA along with the other variables at the enrollment point and 1-year follow-up in our participants (Table 2). 
Table 2

CD4+, CD8+, immune activation and HIV reservoir markers

\begin{tabular}{|c|c|c|c|c|c|c|}
\hline \multirow[t]{2}{*}{ Measure } & \multicolumn{2}{|c|}{ Enrollment } & \multicolumn{2}{|c|}{ Follow-up } & \multirow[t]{2}{*}{$\mathrm{N}=$} & \multirow[t]{2}{*}{$P *$} \\
\hline & Median & IQR & Median & IQR & & \\
\hline CD4 + CD38+ (\%) & 3.2 & $1.68-5.03$ & 2.99 & $2.1-4.61$ & 29 & .754 \\
\hline CD4 + Ki-67+ (\%) & 5.43 & $3.64-7.71$ & 5.13 & $3.82-6.32$ & 29 & .417 \\
\hline CD8 + CD38+ (\%) & 0.82 & $0.43-2.08$ & 3.25 & $2.5-4.6$ & 29 & $<.001$ \\
\hline CD8 + Ki-67+ (\%) & 4.37 & $2.19-5.27$ & 2.66 & $2.15-4.17$ & 29 & .194 \\
\hline IL-7 (pg/ul) & 13.35 & $9.15-16.95$ & 10.43 & $8.23-13.48$ & 29 & .094 \\
\hline Proviral DNA $\left(\log _{10} \mathrm{C} / 10^{6}\right)$ & 2.66 & $2.4-3.44$ & 3.18 & $2.77-3.36$ & 29 & .003 \\
\hline $\begin{array}{l}\text { 2-LTR circles, } n\left(\log _{10}\right. \\
\left.\mathrm{c} / 10^{6}\right)\end{array}$ & $\begin{array}{l}12 \\
(1.47)\end{array}$ & $\begin{array}{l}12(1.31- \\
1.67)\end{array}$ & $\begin{array}{l}13 \\
(1.63)\end{array}$ & $\begin{array}{l}13(1.44- \\
1.84)\end{array}$ & 29 & $.372^{\star \star}$ \\
\hline $\mathrm{CD} 4+\left(\mathrm{cell} / \mathrm{mm}^{3}\right)$ & 546 & $364.5-679.5$ & 566 & $402-720$ & 29 & .424 \\
\hline $\mathrm{CD} 8+\left(\mathrm{cell} / \mathrm{mm}^{3}\right)$ & 765 & $592.5-909$ & 757 & $628.5-902$ & 29 & .966 \\
\hline CD4/CD8 ratio & 0.73 & $0.73-0.99$ & 0.82 & $0.51-0.99$ & 29 & .114 \\
\hline \multicolumn{7}{|c|}{ IQR, interquartile range; $N=29$. } \\
\hline \multicolumn{7}{|c|}{ * Related samples Wilcoxon signed rank test. } \\
\hline
\end{tabular}

As shown in Fig. 1D and 1E, we found a positive correlation between the pre-treatment RNA viral load and the proviral DNA at enrollment $(r=.216, P=.260)$ and at 1-year follow-up $(r=.208, P=.277)$. Although these correlations were not statistically significant, they were consistent and the trend suggests that the size of the reservoir in our chronically infected participants was still influenced by the pre-treatment viraemia, despite many years of suppressive cART. Conversely, there was no significant correlation between the proviral DNA and the nadir CD4 + T-cell count at enrollment and at 1-year follow-up $(r=-.065$, $P=.739 ; r=.048, P=.804)$.

Based on the evidence that has demonstrated a slow decay of the reservoir overtime, we investigated the correlation between the proviral DNA and the number of years that the participants were on CART. We found a very weak and not statistically significant correlation $(r=.141, P=.465$ at enrollment; $r=.148, P$ $=.442$ at follow-up). Furthermore, we investigated the levels of the proviral DNA by subgroups of participants with $\leq 10$ and $>10$ years under CART, but they were similar at enrollment (2.6 versus 2.8 $\log _{10}$ copies $/ 10^{6}, P=.976$ ) and at follow-up (3.1 versus $3.3 \log _{10}$ copies $/ 10^{6}, P=.429$ ). 
For the analysis of sub-groups by third-component of cART, NNRTI vs PI, there were only 3 participants in the latter group. Still, no significant differences were observed between these sub-groups at the observed points (data not shown).

To examine the presence of ongoing viral replication in the clinical conditions and undetectable viraemia of our participants, we investigated the presence of 2-LTR circles. We were able to detect 2-LTR circles in 12 and 13 participants at enrollment (1.47 $\log _{10}$ copies/106; IQR, 1.31-1.67 $\log _{10}$ copies/10 ${ }^{6}$ ) and at 1year follow-up (1.63 $\log _{10}$ copies/106; IQR, 1.44-1.84 $\log _{10}$ copies/106), respectively. The participants with detectable 2-LTR circles at enrollment were not the same that the ones at follow-up. Some of those became undetectable while others became detectable. The correlation between the levels of the proviral DNA load and the 2-LTR circles load was not statistically significant for both observed points $(r=.21$; $P$ $=.274$ at enrollment; and $r=-.124, P=.521$ at follow-up).

\section{CD4 + and CD8 + T-cell activation}

The prospective analysis revealed that the proportion of activated CD4 + T-cells (expressing CD38+), remained without statistically significant changes when comparing both observed points $(P=.754) ; 3.2 \%$ (3.34\%; IQR, 1.69-5.03\%) and 2.99\% (2.55\%; IQR, 2.07-4.61\%) at enrollment and follow-up, respectively (Fig. 2A).

Remarkably, we found a significant increase of the levels in CD8 + T-cell activation at follow-up as compared to those at the enrollment observations $(0.82 \%$ versus $3.25 \%$; $P<.001)$, as shown in Fig. $2 \mathrm{~A}$. Furthermore, in the sub-group analysis, we also found higher levels of CD $8+T$-cell activation at follow-up than those at enrollment. This increase was significant for the participants with $\leq 10$ years of viral suppression $(P=.003)($ Fig. 3A).

To study the increasing levels of immune activation through time, we next examined the correlation between the levels of T-cell activation and the time that the participants have been on CART. Interestingly, we found a positive correlation between the time that the participant had been under CART and the proportion of $\mathrm{CD} 4+\mathrm{T}$-cell activation $(r=.399, \mathrm{P}=.032)(\mathrm{Fig} .4 \mathrm{~A})$. Equally, we observed a similar correlation for the CD8 + T-cell activation, although this was not statistically significant $(r=.206, P=.283)$ (Fig. 4B).

\section{Correlation between the reservoir and the levels of T-cell activation}

To investigate the influence of the reservoir on the immune activation levels, we sought to measure the correlation between the size of the reservoir and the immune activation markers. As shown in Fig. 5, the correlations that we found between the proviral DNA load and both the CD4 + and CD8 + T-cell activation levels were very low and not statistically significant.

Likewise, the correlation between the proportion of T-cell activation and the 2-LTR load was not significant (data is not shown). Remarkably, we found converse proportions of activated CD $4+$ T-cells in subjects 
who had undetectable versus those with detectable 2-LTR circles at enrollment (3.73\% versus $2.04 \%, \mathrm{P}$ $=.03)$ compared to those at follow-up $(2.74 \%$ versus $3.75 \%, P=.682)$, respectively.

\section{Ki-67 + proliferation marker}

The proportion of T-cells that were expressing Ki- $67+$ declined at follow-up for both CD $4+$ and CD $8+T$ cell subsets when compared to those at the enrollment, but remained without statistically significant differences ( $5.43 \%$ versus $5.13 \%, P=.417$ for $C D 4+$; and $4.37 \%$ versus $2.66 \%, P=.194$ for CD $8+$ ) (Fig. $2 B$ ). Despite the significant increase of the CD4 + T-cell count, no significant correlation was observed between CD4 + Ki-67 + T-cells and the nadir CD4 + T-cell count $(r=.049, P=.802$ at enrollment; and $r=-.193, P$ $=.316$ at follow-up). Furthermore, we did not found significant differences when we compared the Ki- $67+$ expression between groups of time under suppressive cART ( $\leq 10$ versus $>10$ years) (Fig. 3B).

To examine the link between proliferation and T-cell activation, we investigated the correlation between CD38 + and Ki-67 + T-cells among CD $4+$ and CD8 + T-cell subsets. In this analysis, we found a positive correlation that was stronger at follow-up compared to that at the enrollment in CD4 + T-cells $(r=.008, P$ $=.966$ at enrollment; and $r=.329, \mathrm{P}=.082$ at follow-up), but remained very similar in CD8 $+\mathrm{T}$-cells $(\mathrm{r}$ $=.327, \mathrm{P}=.084$ at enrollment; and $\mathrm{r}=.339, \mathrm{P}=.072$ at follow-up).

We next investigated the correlation between the proviral DNA and 2-LTR loads with the proportion of CD4 + T-cells expressing Ki-67+, to test if the size of the reservoir was associated with the levels of T-cell proliferation in our participants. Nonetheless, the correlation between the proviral DNA load and the proportion of CD4 + Ki-67 + T-cell was not statistically significant in the analysis at enrollment $(r=-.309$, $\mathrm{P}=.103)$ and neither at follow-up $(\mathrm{r}=.181, \mathrm{P}=.348)$. Interestingly, we found a higher proportion of CD4 + $\mathrm{Ki}-67+\mathrm{T}$-cells in the group of participants in which we observed an increase of the proviral DNA load at follow-up, as compared to that in the group in which it declined $(5.24 \%$ versus $3.83 \%, P=.636)$.

Correspondingly, there was no clear correlation between the proportion of proliferating CD4 + Ki-67+ Tcells and the 2-LTR circles load ( $r=.007, \mathrm{P}=.972$ at enrollment; and $r=-.167, \mathrm{P}=.386$ at follow-up).

Additionally, the proportion of CD4 + Ki-67 + T-cells was higher in the participants who had undetectable versus detectable 2 -LTR circles at enrollment $(5.45 \%$ versus $4.98, P=.679)$ and at follow-up $(5.42 \%$ versus $4.32 \%, P=.398)$.

\section{IL-7}

IL-7 has an important role in T-cell homeostasis and has been used to boost the increase of the CD $4+\mathrm{T}$ cell count in HIV positive subjects. Also, in vivo stimulation with IL-7 has shown to promote the increase of the absolute number of CD4 + T-cells harbouring integrated HIV DNA $(19,20)$.

To investigate if the levels of T-cell immune activation were associated with a proliferative stimulus, we measured the levels of plasmatic IL-7 at both observed points. As shown in Table 2, we found a slight decay in the levels of plasmatic IL-7 after 1-year follow-up, but without a significant variation $(P=.094)$. 
Although the differences were not statistically significant, IL-7 levels were higher in those participants with $\leq 10$ versus those with $>10$ years under suppressive cART (13.8 versus $9.8 \mathrm{pg} / \mu \mathrm{l}, \mathrm{P}=.181$ at enrollment; and 11.7 versus $9.2 \mathrm{pg} / \mu \mathrm{l}, \mathrm{P}=.279$ at follow-up). Remarkably, there was a positive correlation between the proportion of CD $4+\mathrm{CD} 38+\mathrm{T}$-cell and the levels of plasmatic IL-7 at the enrollment point $(\mathrm{r}$ $=.41, \mathrm{P}=.834)$, but not at 1-year follow-up $(r=-.284, \mathrm{P}=.135)$.

Likewise, we found a significant positive correlation between the levels of IL-7 and the proportion of CD4 $+\mathrm{Ki}-67+\mathrm{T}$-cell at enrollment $(r=.52, \mathrm{P}=.004)$, but not at 1-year follow-up $(r=-.136, \mathrm{P}=.482)$.

\section{Discussion}

The origin of the abnormally high levels of immune activation persisting overtime in HIV positive individuals remains unclear. Under the hypothesis of residual low-level viral replication originating from the HIV reservoir, we aimed to investigate the correlation between the levels of immune activation and the size of the reservoir.

In our cohort of patients undergoing long-term effective CART (as determined by the significant recovery of the CD4 + T-cell count, the absence of blips and documented comorbidities) we observed a positive correlation between the CD4 + T-cell activation and the time under CART at 1-year of follow-up, as well as a significant increase of the levels of CD8 + T-cell activation.

In the present study, we found a very weak correlation of the proviral DNA and 2-LTR circles loads with the levels of T-cell activation and proliferation. Studies have described stronger correlations of the proviral DNA during the early stages of infection $(21,22)$. Furthermore, it has been described a progressive reduction of the proviral DNA overtime after the primary infection and thus, it is possible that any previous stronger correlation in our participants could have been lost at some point during their treatment history. Unfortunately, we cannot explore this hypothesis due to the lack of available retrospective samples in our cohort. Still, we believe that this might be the case of our participants, since they have been under a median of 8 years under suppressive CART. In support of this hypothesis, a prospective study has documented the loss of a similar foregoing correlation between the reservoir and immune activation after 4 years of cART in a cohort of HIV positive subjects (23).

On the other hand, the absence of a strong correlation with the proviral DNA in our participants is relevant since we found increasing levels of immune activation (predominantly in CD8 + T-cell), which suggest ongoing indirect or independent mechanisms to the reservoir are influencing immune activation in the long term, despite chronic cART, and the absence of clinical evidence of replication and comorbidities. In this regard, the absence of blips was important for excluding potential viral replication coming from the reservoir under the real-life clinical settings of HIV positive patients. Furthermore, a progressive increase of CD $8+C D 38+T$-cell can be concerning and becomes clinically relevant since higher levels have been associated with higher risk of virological failure, increased morbidity and early mortality $(11,24-28)$. It would be noteworthy to further investigate the presence of other markers in subjects with even more years on CART than our participants (with increasing immune activation and even in the presence of 
associated morbidity), to identify underlying mechanisms driving immune activation other than the HIV reservoir and residual viral replication by themselves.

The stable presence of the reservoir was evidenced by the steady levels of the proviral DNA (in subjects with $\leq 10$ versus those with $>10$ years of $C A R T$ ) and the lack of correlation with the number of years under CART.

Of note, our findings revealed a consistent positive correlation between the HIV proviral DNA and the levels of pre-treatment viral load, highlighting the influence of the viraemia for the establishment of the reservoir (even after at least 5 years of cART), and the critical role that early initiation of cART will have in future HIV cure trials.

In the present study, we were able to detect 2-LTR circles without overall significant variations over the period of 1 year. Though there was no correlation of the 2-LTR circles with the proviral DNA nor with the immune activation, we consider that the variations of the 2-LTR circles within the participants show that some underlying ongoing viral activity might be present, however, its impact is not yet clear.

The stable levels of T-cells expressing Ki-67 + overtime, and the lack of correlation with the levels of immune activation, indicate that the expression of immune activation markers is not entirely associated with the proliferative activity of T-cells. This suggest that other factors might be stimulating the expression of immune activation markers on T-cells. Of note, we observed that the proportion of T-cells expressing Ki-67 + was higher in the participants in which we observed an increase of the proviral DNA load at follow-up. Although we did not find a correlation between Ki-67+ and the proviral load, we believe that the study of the role of cell to cell HIV transmission and clonal expansion of infected cells could provide a more comprehensive view of the dynamics of the reservoir in the context of chronic effective CART and immune activation.

In HIV positive patients IL-7 can be found in higher levels than in negative subjects, which has been explained as a homeostatic effort to restore the T-cell count. Furthermore, the decrease of the plasmatic levels of IL-7 has been associated with the increase of the T-cell count in HIV positive patients (29). In our cohort, we documented lower IL-7 plasmatic levels at the follow-up observation. Remarkably, we found lower levels in the group of subjects with $>10$ years on cART. However, we do not find a correlation with the T-cell count, nor with the levels of immune activation. Interestingly, we found a significant positive correlation at the enrollment point between CD4 + Ki-67 + T-cells and the levels of IL-7, that was lost at the follow-up.

\section{Limitations}

We acknowledge some limitations of the present study. We quantified the proviral DNA and 2-LTR circles from PBMCs as markers of the reservoir, which do not necessarily reflect the number of cells with replication-competent virus that can be found in the actual anatomical reservoirs. However, PBMCs have been effectively used in previous studies to extrapolate the size of the reservoir, given the difficulty to 
obtain cells directly from the lymph nodes in human subjects. On the other hand, this approach also limited the number of available cells to investigate the impact of the reservoir present in specific T-cell sub-populations.

We are aware that DNA PCR methods detect all HIV genomes irrespectively of whether they are fully infectious or not. Defective proviruses may still be producing viral antigens, stimulating the immune system. However, there is still no consensus about the technique for the exact quantification of the reservoir.

Also, we do not exclude the potential presence of underlying undiagnosed conditions in some of our participants. However, we believe that the characteristics and settings of our participants (suppressive chronic cART, the absence of blips, the significant recovery of the CD $4+$ T-cell count and the lack of clinical evidence of other diseases) reduce the probability of major bias.

\section{Conclusion}

The findings from this study do not support the existence of a strong correlation of the proviral DNA and 2-LTR circles load with the levels of immune activation in PBMCs of HIV-1 positive subjects under suppressive chronic cART settings. Still, we do not rule out the possible influence of residual replication from replication-competent T-cells on anatomical reservoirs. It will be challenging and important to further investigate if the reservoir in the lymph nodes (the principal source of the most virally competent virus after CART discontinuation) are responsible for the abnormally high immune activation levels in virally suppressed subjects. Also, in the absence of detectable viral replication, the role of cell to cell transmission and T-cell expansion of infected cells influencing immune activation should be examined.

At this point, in the absence of viral replication in chronic subjects on cART, we believe that other unknown immunological mechanisms driving immune activation are likely involved, which might have been triggered since the early stages of the infection.

Even after long-term suppressive cART (at least 5 years without any blips) we were able to quantify the proviral DNA and to detect 2-LTR circles from PBMCs. In our study, the pre-treatment RNA viral load appeared to correlate with the size of the reservoir. This finding supports the critical role of the acute viraemia for the establishment of the HIV reservoir and the importance of initiating cART as early as possible anticipating future eradication trials.

After our data, we believe that in homeostatic conditions (other than CART) in chronic patients, IL-7 is not likely to be a determinant factor involved with the increase of immune activation solely. However, we believe that the potential of modulating IL-7 in vivo should be tested given the evidence from previous studies.

Most importantly, we highlight the presence of increasing levels of immune activation overtime (especially in CD8 + T-cells), which is concerning in the context of viral suppression, CD4 + T-cell count 
recovery and the absence of other risk factors for its future impact on the morbidity of patients in the long term, a subject that should be further investigated and addressed.

In summary, our study supports the important role of early CART in limiting the reservoir, the limited role of the reservoir influencing the increasing levels of immune activation in the context of chronic effective CART and the need to further investigate underlying origins and immunological pathways for the design of effective interventions, especially in the era of chronically treated patients.

\section{Declarations}

\section{Ethics approval and consent to participate}

This study was approved by the research and bioethics boards of the INCMNSZ (REF. 1783) and was conducted in accordance with the Helsinki Declaration. All participants provided written informed consent prior to participation in this study.

\section{Consent for publication}

Not applicable.

\section{Availability of data and materials}

Complete materials and methods can be found in the supplementary file.

\section{Competing interests}

The authors declare that they have no competing interests.

\section{Funding}

This study was financially supported by the Fondo Sectorial de Investigación en Salud y Seguridad Social [Grant no. FOSISS-2015-262426] from Consejo Nacional de Ciencia y Tecnología (CONACYT) of Mexico.

\section{Authors' contributions}


AOR: Writing - original draft, review and editing, enrollment of study participants, PBMCs isolation, staining for flow-cytometry, flow-cytometry data analysis, IL-7 ELISA assays and statistical analysis. MVR: Draft review and editing, flow-cytometry protocol standardization, PBMCs isolation, staining for flow-cytometry and IL-7 ELISA assays. LLFR: Standardization protocols, HIV proviral DNA and 2-LTR circles viral load assays, PBMCs isolation and staining for flow-cytometry. MVM: HIV proviral DNA and 2LTR circles protocols standardization. DPRR: Design of the flow-cytometry original protocol and data analysis standardization. MML: Enrollment of study subjects and obtention of blood samples. MLZD: Enrollment of study participants, PBMCs isolation and quantification. EYVL: PBMCs isolation. RARD: Laboratory consultant and critical reviewer. LESR: Original concept and study design, scientific approach, writing - editing and critical review of the manuscript. All authors read and approved the final manuscript.

\section{Acknowledgements}

The authors would like to thank the study participants and the Molecular Virology Unit team of the Instituto Nacional de Ciencias Médicas y Nutrición Salvador Zubirán in Mexico City.

\section{Abbreviations}

2-LTR: 2-Long Terminal repeats (LTR)

AIDS: Acquired Immune Deficiency Syndrome

cART: Combined antiretroviral therapy

DNA: Deoxyribonucleic acid

ELISA: Enzyme-linked immunosorbent assay

HCV: Hepatitis C virus

HIV: Human immunodeficiency virus (HIV) type 1

IL-7: Interleukin 7

INCMNSZ: Instituto Nacional de Ciencias Médicas y Nutrición Salvador Zubirán

IQR: Interquartile range

NNRTI: Non-nucleoside reverse transcriptase inhibitor 
PBMCs: Peripheral blood mononuclear cells

PCR: Polymerase chain reaction

PI: Protease inhibitor

RNA: Ribonucleic acid

\section{References}

1. Deeks SG, Tracy R, Douek DC. Systemic effects of inflammation on health during chronic HIV infection. Immunity. 2013 Oct 17;39(4):633-45.

2. Wada NI, Jacobson LP, Margolick JB, Breen EC, Macatangay B, Penugonda S, et al. The effect of HAART-induced HIV suppression on circulating markers of inflammation and immune activation. AIDS. 2015 Feb 20;29(4):463-71.

3. Utay NS, Hunt PW. Role of immune activation in progression to AIDS. Curr Opin HIV AIDS. 2016 Mar;11(2):131-7.

4. Lederman MM, Funderburg NT, Sekaly RP, Klatt NR, Hunt PW. Residual immune dysregulation syndrome in treated HIV infection. Adv Immunol. 2013;119:51-83.

5. Ipp H, Zemlin AE, Erasmus RT, Glashoff RH. Role of inflammation in HIV-1 disease progression and prognosis. Crit Rev Clin Lab Sci. 2014 Apr;51(2):98-111.

6. Krebs SJ, Ananworanich J. Immune activation during acute HIV infection and the impact of early antiretroviral therapy. Curr Opin HIV AIDS. 2016 Mar;11(2):163-72.

7. d'Ettorre G, Paiardini M, Zaffiri L, Andreotti M, Ceccarelli G, Rizza C, et al. HIV persistence in the gut mucosa of HIV-infected subjects undergoing antiretroviral therapy correlates with immune activation and increased levels of LPS. Curr HIV Res. 2011 Apr;9(3):148-53.

8. Brenchley JM, Price DA, Schacker TW, Asher TE, Silvestri G, Rao S, et al. Microbial translocation is a cause of systemic immune activation in chronic HIV infection. Nat Med. 2006 Dec;12(12):1365-71.

9. Jiang W, Lederman MM, Hunt P, Sieg SF, Haley K, Rodriguez B, et al. Plasma levels of bacterial DNA correlate with immune activation and the magnitude of immune restoration in persons with antiretroviral-treated HIV infection. J Infect Dis. 2009 Apr 15;199(8):1177-85.

10. Márquez M, Romero-Cores P, Montes-Oca M, Martín-Aspas A, Soto-Cárdenas M-J, Guerrero F, et al. Immune activation response in chronic HIV-infected patients: influence of Hepatitis $\mathrm{C}$ virus coinfection. PLoS One. 2015 Mar 16;10(3):e0119568.

11. Serrano-Villar S, Sainz T, Lee SA, Hunt PW, Sinclair E, Shacklett BL, et al. HIV-infected individuals with low CD4/CD8 ratio despite effective antiretroviral therapy exhibit altered T cell subsets, heightened CD8+ T cell activation, and increased risk of non-AIDS morbidity and mortality. PLoS Pathog. 2014 May 15;10(5):e1004078. 
12. Jain V, Hartogensis W, Bacchetti P, Hunt PW, Hatano H, Sinclair E, et al. Antiretroviral therapy initiated within 6 months of HIV infection is associated with lower T-cell activation and smaller HIV reservoir size. J Infect Dis. 2013 Oct 15;208(8):1202-11.

13. Deeks SG, Kitchen CMR, Liu L, Guo H, Gascon R, Narváez AB, et al. Immune activation set point during early HIV infection predicts subsequent CD4+ T-cell changes independent of viral load. Blood. 2004 Aug 15;104(4):942-7.

14. Hunt PW, Landay AL, Sinclair E, Martinson JA, Hatano H, Emu B, et al. A low T regulatory cell response may contribute to both viral control and generalized immune activation in HIV controllers. PLoS One. 2011 Jan 31;6(1):e15924.

15. Taiwo B, Hunt PW, Gandhi RT, Ellingson A, McKenna M, Jacobson JM, et al. CD8+ T-cell activation in HIV-1-infected patients experiencing transient low-level viremia during antiretroviral therapy. J Acquir Immune Defic Syndr. 2013 May 1;63(1):101-4.

16. Zoufaly A, Kiepe JG, Hertling S, Hüfner A, Degen O, Feldt T, et al. Immune activation despite suppressive highly active antiretroviral therapy is associated with higher risk of viral blips in HIV-1infected individuals. HIV Med. 2014 Sep;15(8):449-57.

17. Li JZ, Etemad B, Ahmed H, Aga E, Bosch RJ, Mellors JW, et al. The size of the expressed HIV reservoir predicts timing of viral rebound after treatment interruption. AIDS. 2016 Jan 28;30(3):343-53.

18. Hill AL, Rosenbloom DIS, Goldstein E, Hanhauser E, Kuritzkes DR, Siliciano RF, et al. Real-Time Predictions of Reservoir Size and Rebound Time during Antiretroviral Therapy Interruption Trials for HIV. PLoS Pathog. 2016 Apr 27;12(4):e1005535.

19. Lévy Y, Sereti I, Tambussi G, Routy JP, Lelièvre JD, Delfraissy JF, et al. Effects of recombinant human interleukin 7 on T-cell recovery and thymic output in HIV-infected patients receiving antiretroviral therapy: results of a phase I/Ila randomized, placebo-controlled, multicenter study. Clin Infect Dis. 2012 Jul;55(2):291-300.

20. Vandergeeten C, Fromentin R, DaFonseca S, Lawani MB, Sereti I, Lederman MM, et al. Interleukin-7 promotes HIV persistence during antiretroviral therapy. Blood. 2013 May 23;121(21):4321-9.

21. Benito JM, López M, Lozano S, Ballesteros C, Martinez P, González-Lahoz J, et al. Differential upregulation of CD38 on different T-cell subsets may influence the ability to reconstitute CD4+ T cells under successful highly active antiretroviral therapy. J Acquir Immune Defic Syndr. 2005 Apr $1 ; 38(4): 373-81$.

22. Ananworanich J, Sacdalan CP, Pinyakorn S, Chomont N, de Souza M, Luekasemsuk T, et al. Virological and immunological characteristics of HIV-infected individuals at the earliest stage of infection. J Virus Erad. 2016;2:43-8.

23. Gandhi RT, McMahon DK, Bosch RJ, Lalama CM, Cyktor JC, Macatangay BJ, et al. Levels of HIV-1 persistence on antiretroviral therapy are not associated with markers of inflammation or activation. PLoS Pathog. 2017 Apr 20;13(4):e1006285.

24. Liu Z, Cumberland WG, Hultin LE, Prince HE, Detels R, Giorgi JV. Elevated CD38 antigen expression on CD8+ T cells is a stronger marker for the risk of chronic HIV disease progression to AIDS and death in 
the Multicenter AIDS Cohort Study than CD4+ cell count, soluble immune activation markers, or combinations of HLA-DR and CD38 expression. J Acquir Immune Defic Syndr Hum Retrovirol. 1997 Oct 1;16(2):83-92.

25. Resino S, Bellón JM, Gurbindo MD, Muñoz-Fernández MA. CD38 expression in CD8+ T cells predicts virological failure in HIV type 1-infected children receiving antiretroviral therapy. Clin Infect Dis. 2004 Feb 1;38(3):412-7.

26. Sherman GG, Scott LE, Galpin JS, Kuhn L, Tiemessen CT, Simmank K, et al. CD38 expression on CD8(+) T cells as a prognostic marker in vertically HIV-infected pediatric patients. Pediatr Res. 2002 Jun;51(6):740-5.

27. Benito JM, López M, Lozano S, Martinez P, González-Lahoz J, Soriano V. CD38 expression on CD8 T lymphocytes as a marker of residual virus replication in chronically HIV-infected patients receiving antiretroviral therapy. AIDS Res Hum Retroviruses. 2004 Feb;20(2):227-33.

28. Tarancon-Diez L, De Pablo-Bernal RS, Álvarez-Rios Al, Rosado-Sánchez I, Dominguez-Molina B, Genebat M, et al. CCR5+ CD8 T-cell levels and monocyte activation precede the onset of acute coronary syndrome in HIV-infected patients on antiretroviral therapy. Thromb Haemost. 2017 Jun 2;117(6):1141-9.

29. Napolitano LA, Grant RM, Deeks SG, Schmidt D, De Rosa SC, Herzenberg LA, et al. Increased production of IL-7 accompanies HIV-1-mediated T-cell depletion: implications for T-cell homeostasis. Nat Med. 2001 Jan;7(1):73-9.

\section{Figures}


A

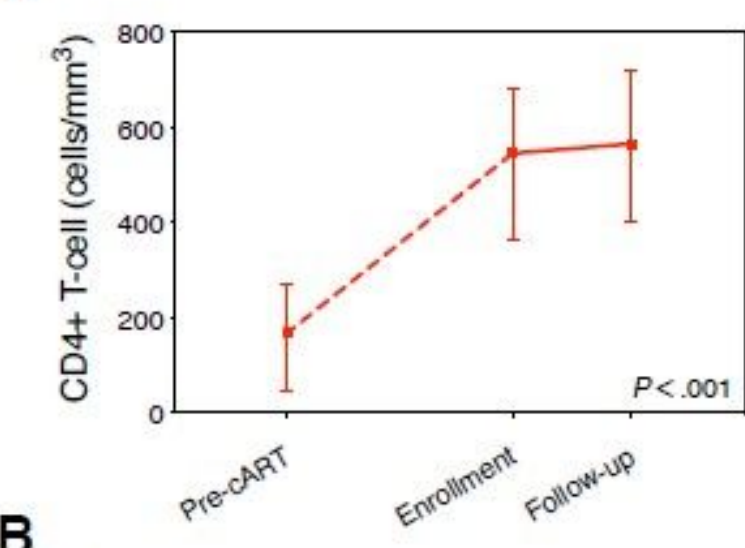

B

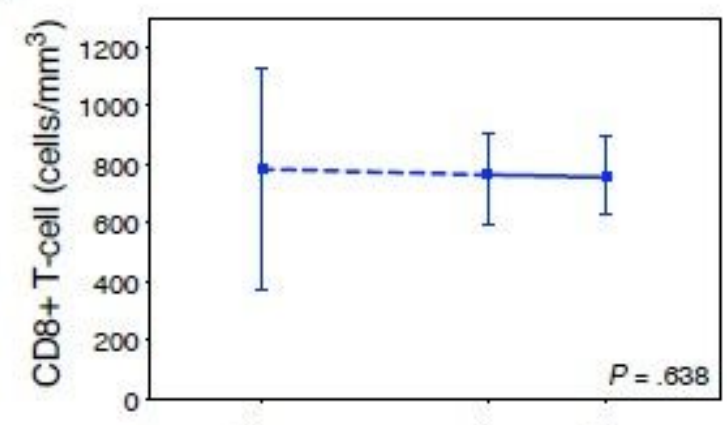

C
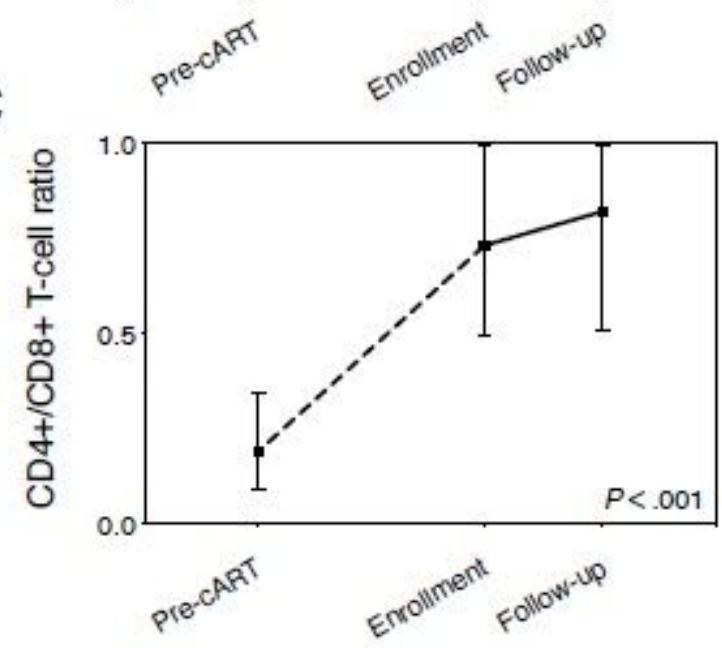

D

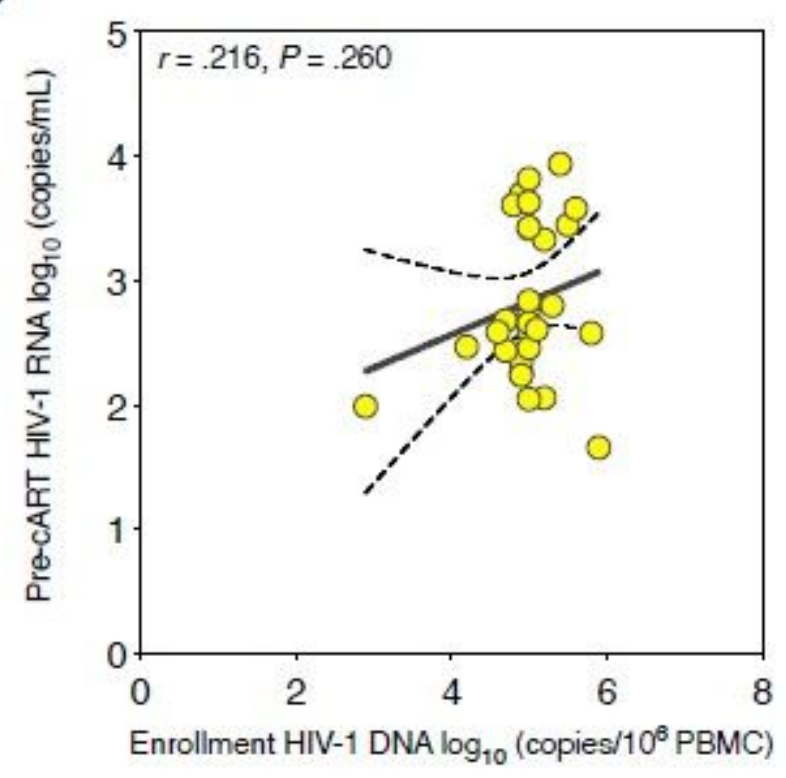

$\mathbf{E}$

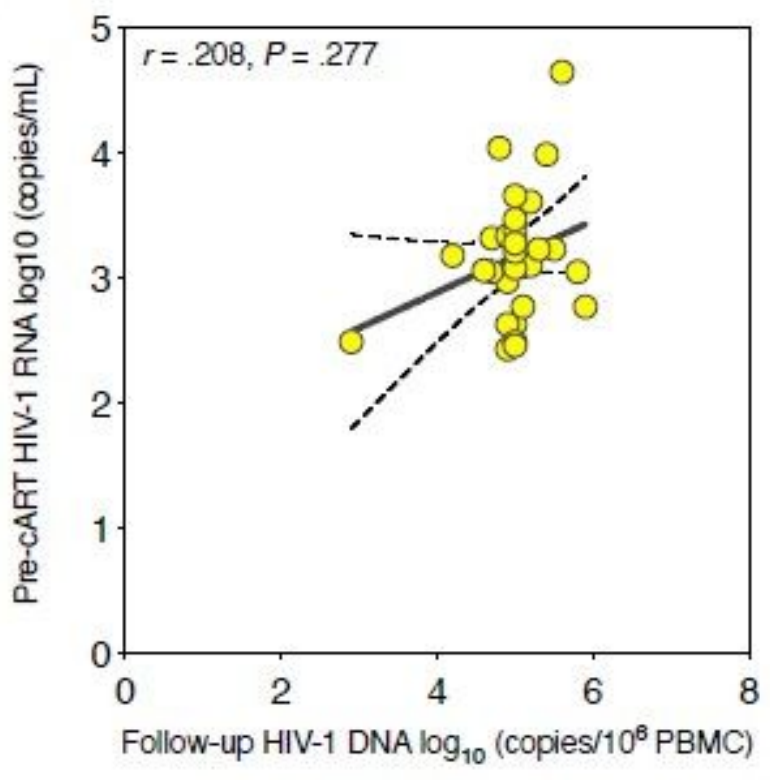

Figure 1

CD4+ and CD8+ T-cell count overtime and the correlation between the viral load and the reservoir The $\mathrm{CD} 4+\mathrm{T}$-cell count significantly recovered after at least 5 years of effective treatment $(P<.001)(A)$. The CD8+ T-cell count remained stable during the follow-up (B), while the CD4/CD8 ratio increased at expenses of the CD4+ T-cell count (C). The positive correlation between the pre-treatment viral RNA load and the proviral DNA load was very consistent at both observed times (Spearman correlation and linear regression analysis [thick lines] with 95\% confidence intervals [dotted lines]) (D, E). 

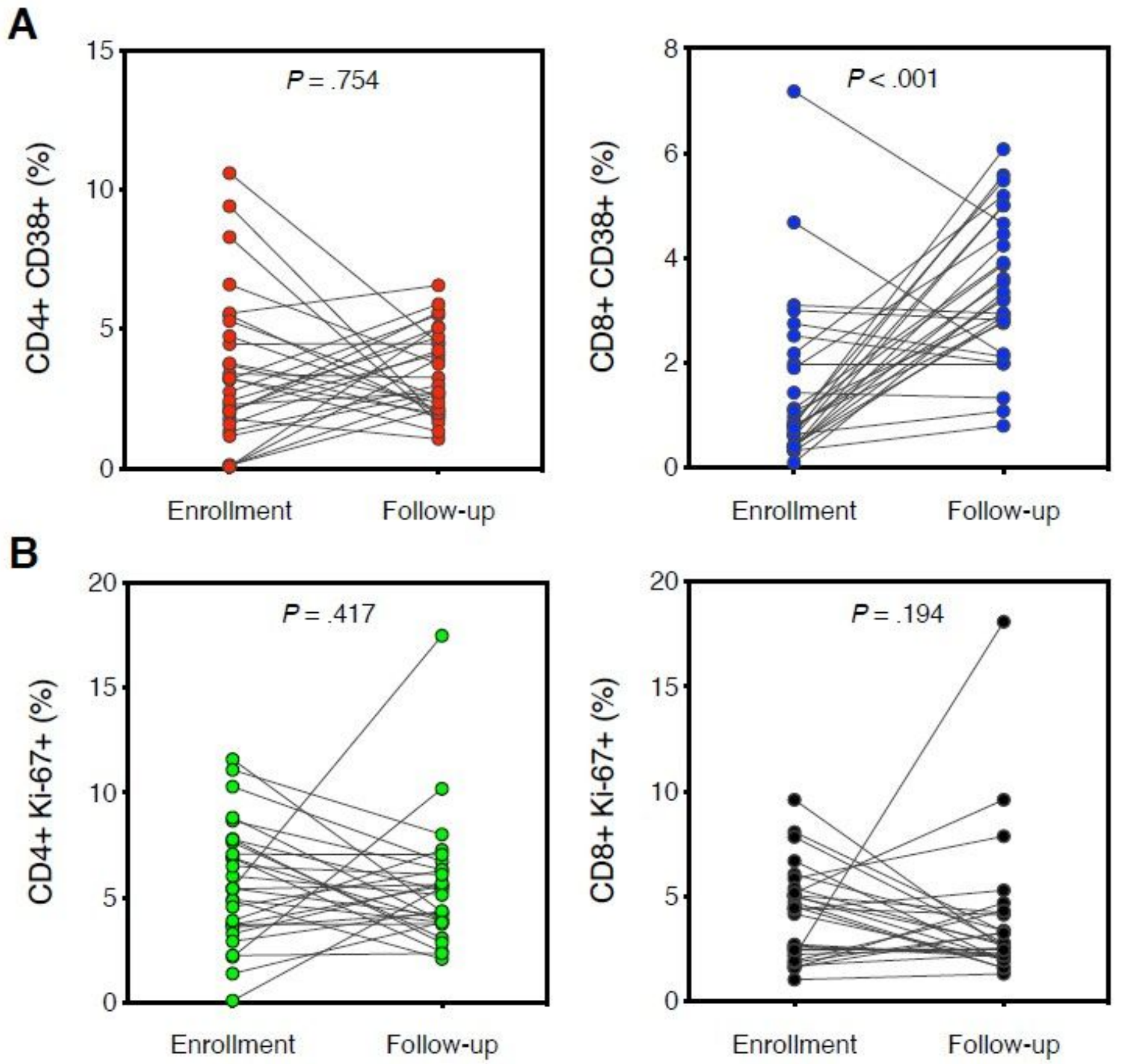

Figure 2

T-cell activation and proliferation levels after 1-year The difference of the levels of expression of CD38+ and Ki-67+ after 1-year follow-up were examined using the Mann-Whitney $U$ test. (A) The left graph shows the levels of CD4+ T-cell activation at enrollment and after 1 year of follow-up. The right graph shows a significant increase in the levels of CD8 T-cell activation at follow-up $(P<.001)$. (B) The left graph shows the levels of CD4+ T-cell proliferation at enrollment and at follow-up, while the right one shows the levels of CD8+ T-cell proliferation at both observed times. In this case, the levels of Ki-67+ remained without significant variations. 
A

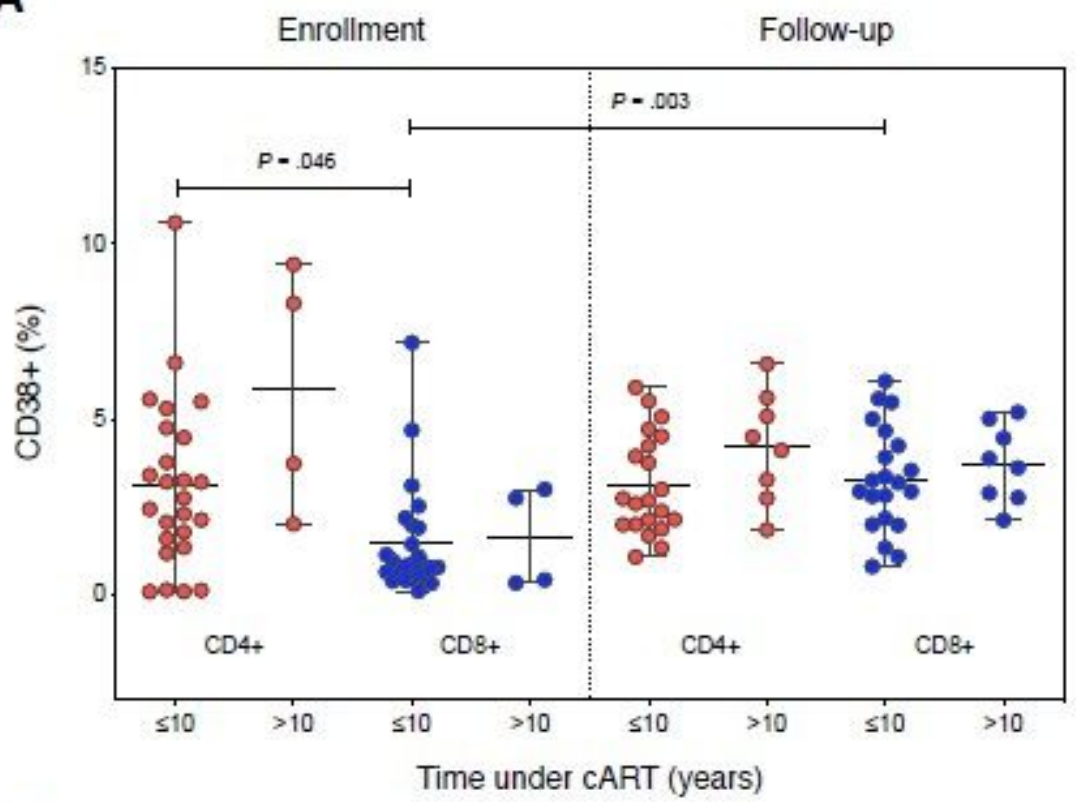

B

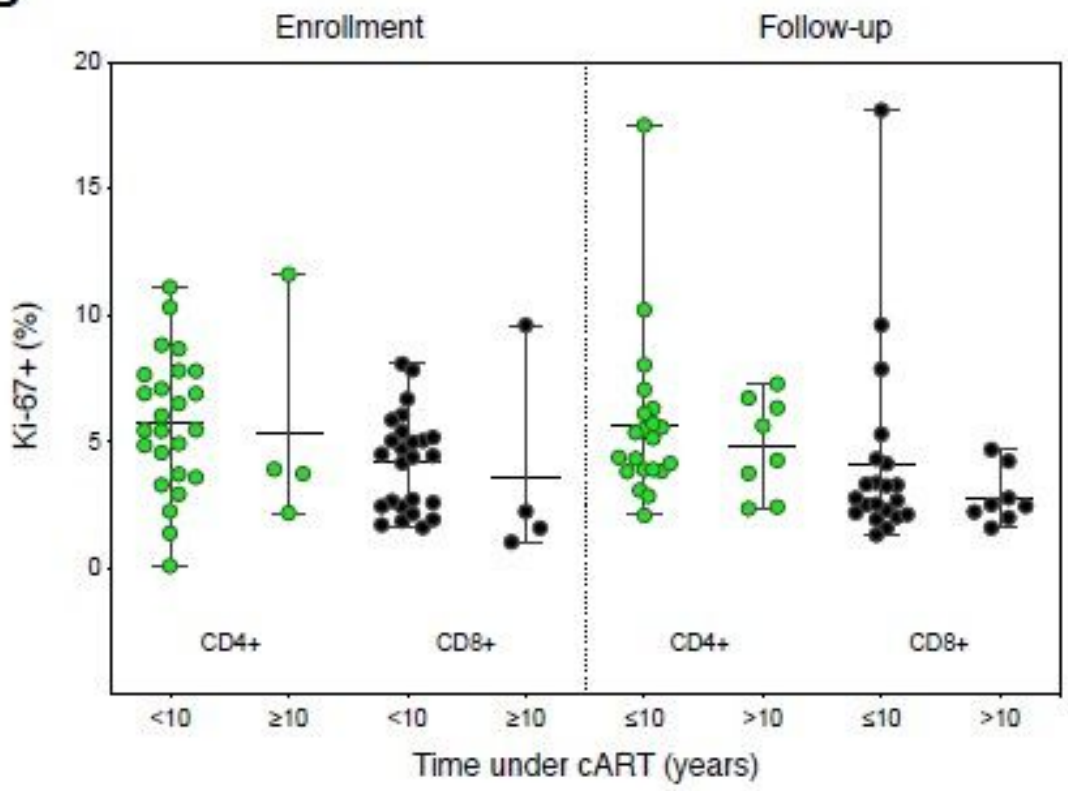

Figure 3

Analysis by groups of time under suppressive treatment and the levels of T-cell activation and proliferation (A) The overall levels of immune activation are higher in the participants with more than 10 years under CART, also, in CD4+ T-cells the levels of activation were higher than CD8+ T-cells. There was a significant increase in the levels of $C D 8+T$-cell activation in the subset with $\leq 10$ years under suppressive CART after 1 year of follow-up ( $P=.003$, Wilcoxon signed-rank test). (B) The levels of expression of Ki-67+ were higher in $\mathrm{CD} 8+\mathrm{T}$-cells compared to $\mathrm{CD} 4+\mathrm{T}$-cells but remained without significant changes among groups. Four subjects moved to the group with $>10$ years at the time of the follow-up visit. 
A

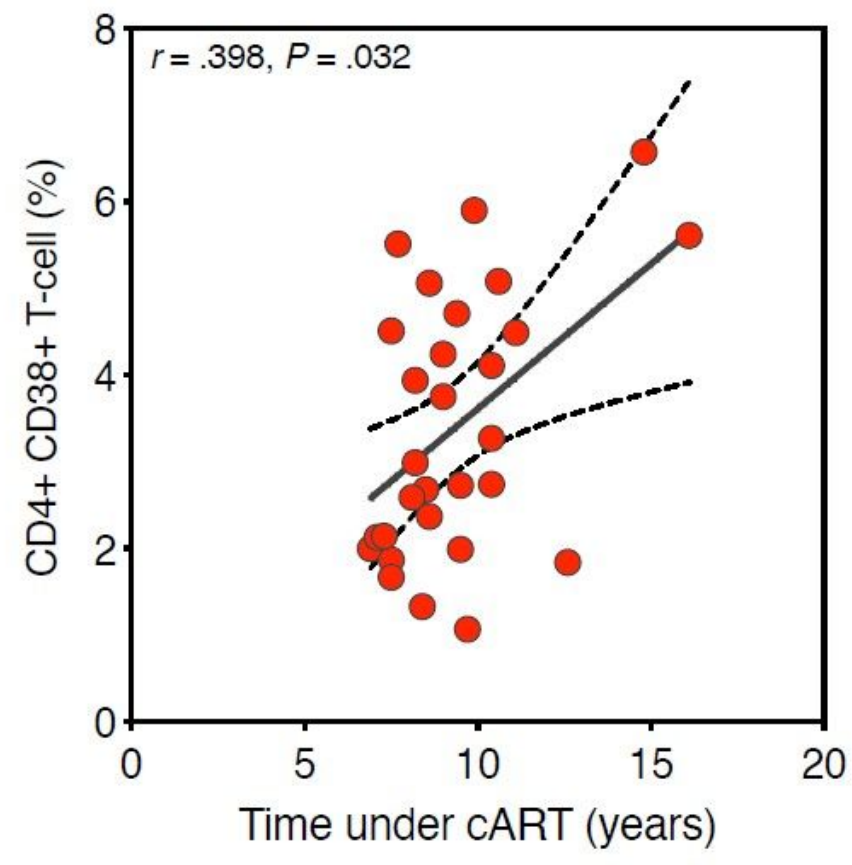

B

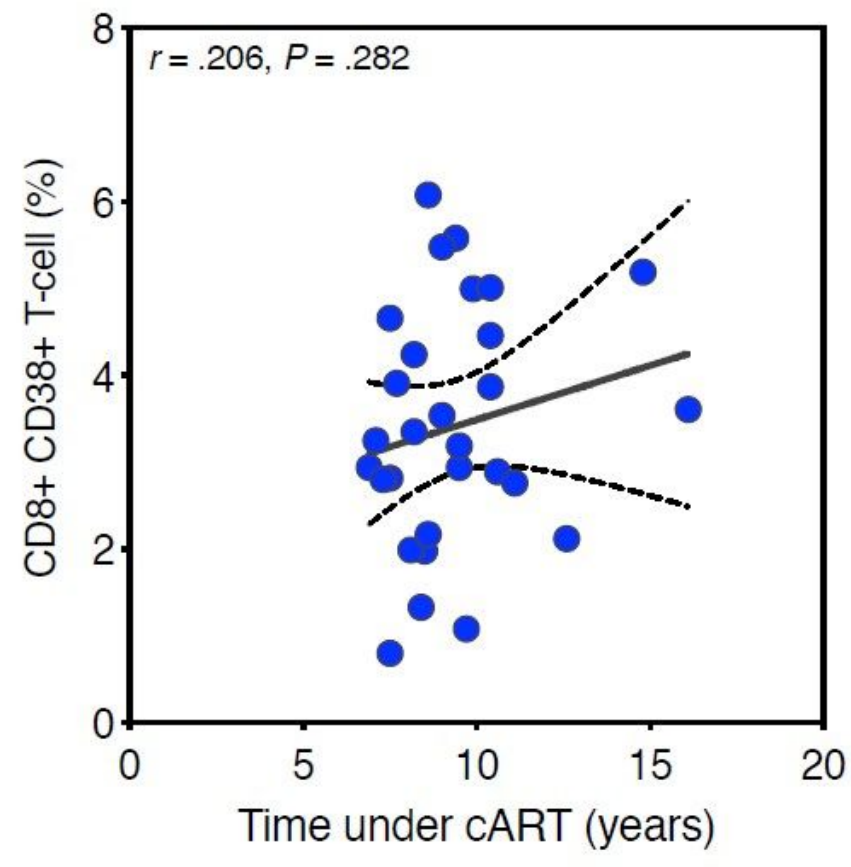

Figure 4

The correlation between the levels of T-cell activation and the time on cART The positive correlation suggest that the levels of immune activation increase overtime during suppressive treatment (Spearman correlation and linear regression analysis [thick lines] with 95\% confidence intervals [dotted lines]). The correlation of the CD4+ T-cell and the time that the subjects have been under CART was stronger and statistically significant $(P=.032)(A)$ when compared to the correlation of $C D 8+T$-cell $(B)$ at the follow-up visit. 
A
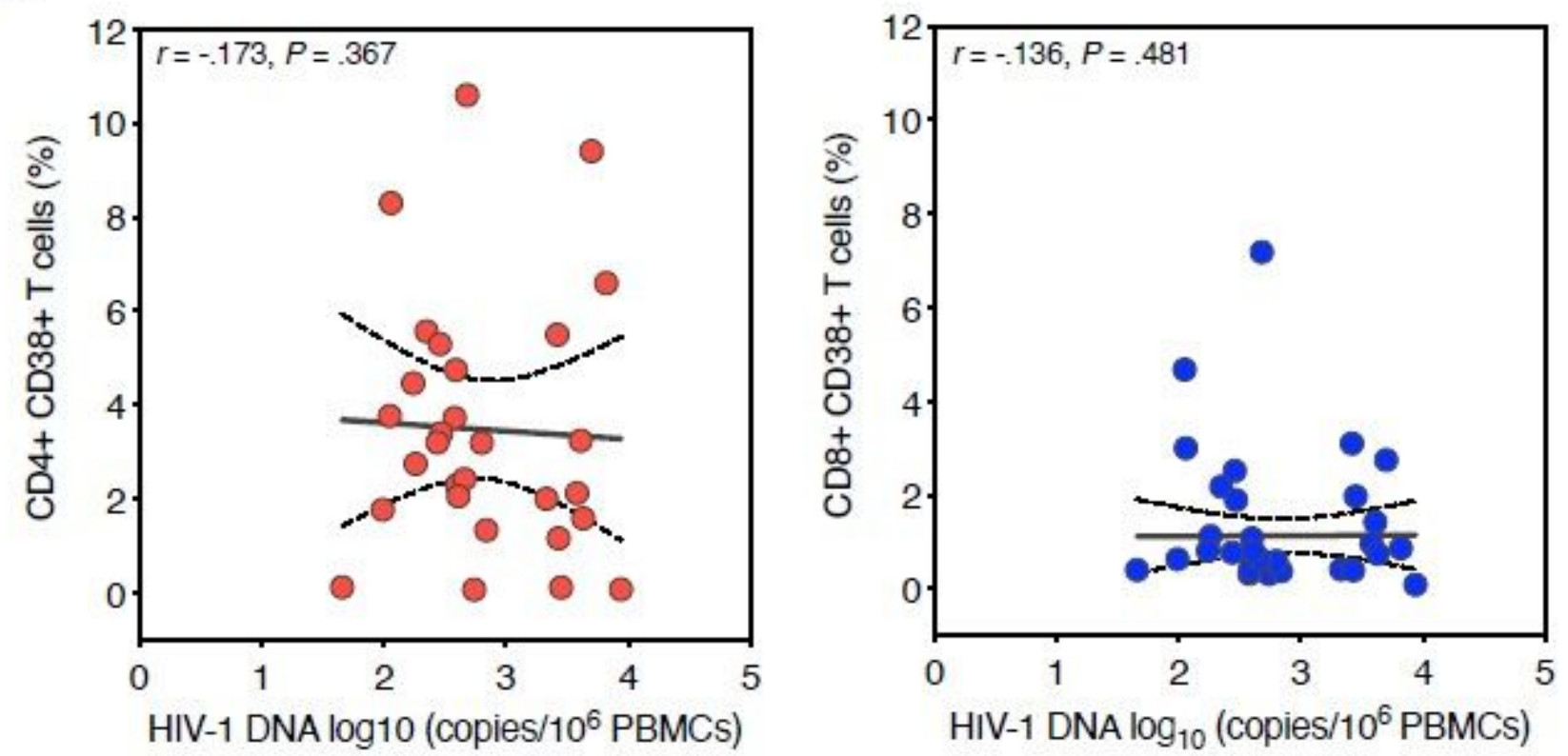

B
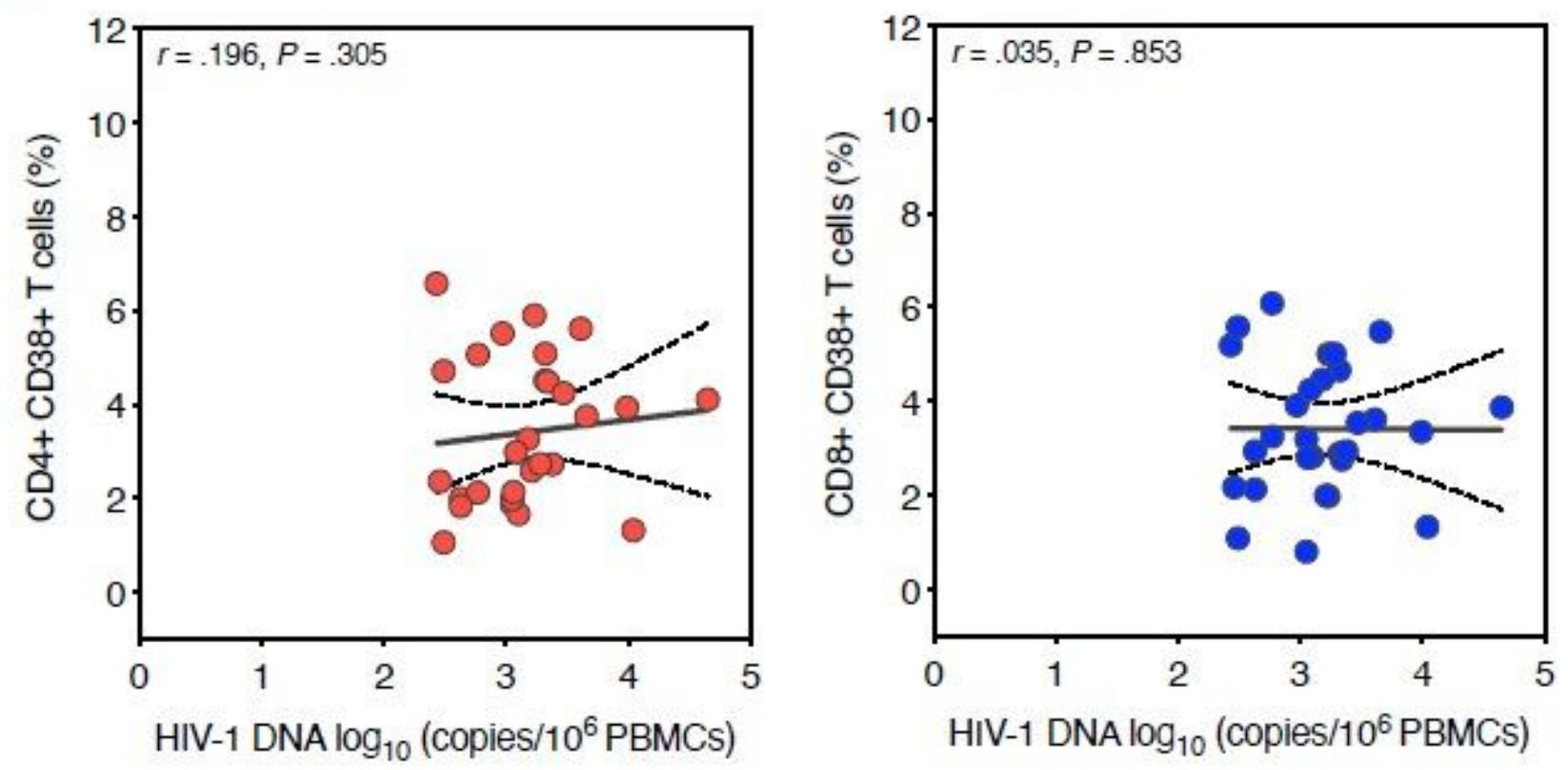

Figure 5

Correlation between the HIV-1 reservoir and the levels of T-cell activation The correlation of CD4+ and CD8+ T-cell activation with the HIV-1 proviral DNA was not strong and neither statistically significant at enrollment (A), and follow-up (B) (Spearman correlation and the linear regression (thick lines) analysis with 95 confidence intervals (dotted lines).

\section{Supplementary Files}

This is a list of supplementary files associated with this preprint. Click to download. 
- Supplementarymaterial.docx

Page 23/23 